\title{
Significant inhibition of infantile hemangioma growth by sustained delivery of urea from liposomes-in-microspheres
}

\author{
XIAOSHUANG ZHU ${ }^{1 *}$, XIAONAN GUO ${ }^{1 *}$, DAKAN LIU $^{1}$, YUBIN GONG $^{1}$, JIN SUN $^{2}$ and CHANGXIAN DONG ${ }^{1}$ \\ ${ }^{1}$ Department of Hemangioma and Vascular Malformation, Henan Provincial People's Hospital, Zhengzhou, Henan 450003; \\ ${ }^{2}$ Department of Pharmacy, Second Military Medical University, Shanghai 200433, P.R. China
}

Received May 26, 2017; Accepted November 1, 2017

DOI: $10.3892 /$ or.2017.6103

\begin{abstract}
Infantile hemangioma (IH) is a benign pediatric tumor, and rapid growth of IH can result in serious morbidity and even mortality. Only one drug Hemangeol ${ }^{\mathrm{TM}}$ (propranolol hydrochloride oral solution) has been approved for the treatment of IH, whereas patients suffer from its adverse effects and high frequency of administration. We have used urea, an organic compound and a normal body metabolite, in the treatment of $\mathrm{IH}$ for 20 years, and demonstrated that urea is an effective and well-tolerated treatment for IH. To reduce the daily administration of urea, we firstly utilized urea-loaded liposomes-in-microspheres (ULIM) as a novel topical controlled release system to realize the sustained release of urea. ULIM were fabricated from the encapsulation of urea-loaded liposomes in poly(lactic-co-glycolic acid)- $b$-poly(ethylene glycol)- $b$-poly(lactic-co-glycolic acid) microspheres. The characteristics, activity and mechanism against IH of ULIM were examined in vitro and in vivo. ULIM were of a desired particle size $(\sim 62.4 \mu \mathrm{m})$, drug encapsulation efficiency $(\sim 51.5 \%)$ and sustained drug release for 40 days. ULIM inhibited the proliferation of hemangioma endothelia cells (HemECs) and expression of vascular endothelial growth factor-A in HemECs. The therapeutic effect of ULIM in IH was better than propranolol, urea, urea-loaded liposomes and urea-loaded microspheres in vivo, as reflected by markedly decreased hemangioma weight, volume and microvessel density. None of the treated mice showed behavioral changes, severe side-effects and weight loss. Our results suggest that use of ULIM is a potential and safe approach with which to
\end{abstract}

Correspondence to: Dr Xiaoshuang Zhu or Dr Changxian Dong, Department of Hemangioma and Vascular Malformation, Henan Provincial People's Hospital, 7 Weiwu Road, Zhengzhou, Henan 450003, P.R. China

E-mail: 15738309990@163.com

E-mail: dong_changxian@yahoo.com

*Contributed equally

Key words: hemangioma, urea, microspheres, liposomes, controlled release locally and efficiently deliver urea to hemangioma, and is a promising alternative to propranolol in the treatment of $\mathrm{IH}$.

\section{Introduction}

Infantile hemangioma (IH) affects approximately 4-10\% of infants, and induces serious morbidity and mortality $(1,2)$. Although Hemangeol ${ }^{\mathrm{TM}}$ (propranolol hydrochloride oral solution), the first US Food and Drug Administration (FDA) approved drug for $\mathrm{IH}$, is effective, its adverse effects include aggravated respiratory tract infections $(3,4)$. Its twice daily oral administration also reduces the compliance of patients. Thus, it is necessary to develop novel alternative drugs to treat $\mathrm{IH}$.

Urea, a normal body metabolite, is crucial for the metabolism of nitrogenous compounds (5). Significantly, urea is highly soluble in water, and practically non-toxic $\left(\mathrm{LD}_{50}\right.$ is $15 \mathrm{~g} / \mathrm{kg}$ for rats). Urea-containing creams are widely used in various skin diseases (6). Since 1970s, local injection of urea has been used in the treatment of IH only in China (7). In our hospital (Henan Provincial People's Hospital, Zhengzhou, China), local injection of urea has been used in the treatment of IH for 20 years (8-11). In a clinical trial consisting of 869 cases of IH in our hospital during 2006-2009, we demonstrated that $54.6 \%$ of the cases were cured, and $31.4 \%$ were improved after urea injection, without observing severe complications (8). In a clinical trial consisting of 167 cases of IH in our hospital during 2009-2011, we demonstrated that $87.4 \%$ of the cases were cured, and $12.6 \%$ were improved after urea injection, without observing severe complications (10). The mechanisms underlying the therapeutic efficacy of urea against IH include direct cytotoxic effects towards hemangioma cells and protein denaturation $(7,11)$. Since urea is injected topically, its side-effects are rare (7-11). The primary side-effect of urea injection for the treatment of IH is skin irritation, which could disappear spontaneously in 3-5 days, and does not need special treatments (7-11). Thus, urea is expected to be an effective and well-tolerated treatment for IH. Although urea local injection has achieved superior therapeutic effects in the treatment of $\mathrm{IH}$, daily injection significantly reduce the compliance of patients (7-11). Thus, it is urgent to reduce the high frequency of administration of urea in the treatment of IH.

To overcome the daily administration, a topical controlled delivery modality could be used for the treatment of IH. The topical administration could target diseases directly, and 
minimize unpredictable absorption and side-effects $(12,13)$. Furthermore, topical delivery modalities could realize controlled release of drugs, resulting in long-lasting potential therapeutic efficacy, with reduced drug doses and administration frequency (14). Thus, we hypothesized that a topical controlled release systems could decrease the high administration frequency of urea.

Liposomes, featured by their good biocompatibility and long in vivo circulation, have been widely used as a controlled release system (15-17). Nevertheless, since liposomes are soft, liposomal sustained drug release is not good $(18,19)$. The biocompatible poly(lactic-co-glycolic acid) (PLGA) microspheres, which are more rigid, have been commonly used as a controlled delivery system (20). Notably, some researchers have realized that the combination of liposomes and PLGA microspheres (liposomes-in-microspheres, LIM), could exert their advantages and avert their disadvantages $(21,22)$. After being coated with PLGA, the stability and drug loading of liposomes could be improved, since both the polymer and liposomal bilayers could retard drug release. Furthermore, by the existence of the lipids on the surface, the biocompatibility of PLGA microspheres could be improved. It is conceivable that liposomes could still act as drug sustained release reservoirs after the liposomes are released from the microspheres. Several researchers have developed LIM for successful sustained release of various drugs suffered from quick drug release from liposomes $(21,22)$.

To reduce the daily administration of urea, we firstly utilized urea-loaded liposomes-in-microspheres (ULIM) as a novel topical controlled release system to realize the sustained release of urea. ULIM were developed from encapsulating urea-loaded liposomes in microspheres made of poly(lactic-co-glycolic acid)-b-poly(ethylene glycol)-bpoly(lactic-co-glycolic acid) copolymers. The characteristics, activity and mechanism against IH of ULIM were examined in vitro and in vivo.

\section{Materials and methods}

Reagents and cell culture. Poly(lactic-co-glycolic acid)$b$-Poly(ethylene glycol)- $b$-Poly(lactic-co-glycolic acid) copolymers (Mw 11,900, PLGA-PEG-PLGA, 1:1 LA:GA, 70 kDa-4.6 kDa-70 kDa) was purchased from Akina, Inc. (West Lafayette, IN, USA). Hydro soy phosphatidylcholine (HSPC) and cholesterol were provided by Avanti Polar Lipids (Alabaster, AL, USA). Propranolol (hydrochloride salt), urea, poly(vinyl alcohol) (PVA, Mw 30,000-70,000), and chitosan (hydrochloride salt) were provided by Sigma-Aldrich (St. Louis, MO, USA). Matrigel was provided by Becton-Dickinson (Franklin Lakes, NJ, USA). All organic reagents were of analytical grade and purchased from China Sinopharm International Co., Ltd. (Shanghai, China).

The human hemangioma endothelial cells (HemECs) derived from the IH of patients were isolated as previously described $(23,24)$. The present study was approved by the Research Ethics Committee of Henan Provincial People's Hospital (Zhengzhou, China). Written informed consent was obtained from all the patients. All specimens were handled and made anonymous according to the ethical and legal standards. First, IH specimens were obtained in Henan Provincial
People's Hospital, and the clinical diagnosis was confirmed at the Department of Pathology in Henan Provincial People's Hospital. The excised IH specimens were stored in Endothelial Cell Growth Medium-2 (EGM-2; Lonza, Walkersville, MD USA) at $4^{\circ} \mathrm{C}$. Then the specimen was cut and trypsinized by $0.25 \%$ trypsin at $37^{\circ} \mathrm{C}$. Then EGM-2 with $10 \%$ fetal bovine serum (FBS) was added to block the trypsinization. The tissue blocks were inoculated in culture plates coated with gelatin. After inoculation, EGM-2 with 10\% FBS was added to the tissue block, and the culture medium was replaced once every three days. The cells were subcultured at a 1:3 ratio when the cells reached confluence.

Preparation of urea-loaded liposomes-in-microspheres. Urealoaded liposomes were prepared by the reverse evaporization method as previously described (25). Briefly, specific amounts of lipids ( $20 \mu \mathrm{mol}, \mathrm{HSPC}$ :cholesterol $=55: 45$, molar ratios) were dissolved in $3 \mathrm{ml}$ diethyl ether. One milliliter of urea solution $(4 \mathrm{mg} / \mathrm{ml})$ was added to the above lipid solution. The mixture was sonicated for $10 \mathrm{~min}$ to form stable $\mathrm{W} / \mathrm{O}$ (water in oil) emulsion. The diethyl ether was then removed by reduction vaporization at $25^{\circ} \mathrm{C}$. After then, distilled water was added, and the resultant multilamellar liposomes (MLL) were obtained. Unilamellar liposomes (ULL) were obtained by the extrusion of the MLL using a LiposoFast ${ }^{\mathrm{TM}}$ extruder (Avestin, Ottawa, ON, Canada) with 200-nm pore size membranes (Whatman ${ }^{\circledR}$ Nuclepore $^{\mathrm{TM}}$ membrane; Thermo Fisher Scientific) with 10 cycles at $65^{\circ} \mathrm{C}$. Sephadex G-50 Gel filtration separated the ULL from the non-encapsulated urea. For the coating of liposomes with chitosan, $1 \mathrm{ml}$ liposome solution was mixed with $2 \mathrm{ml} 1.5 \%$ chitosan solution and incubated at $4^{\circ} \mathrm{C}$ overnight.

Subsequently, urea-loaded liposomes-in-microspheres (ULIM) were prepared. After urea-loaded liposome solution $(0.5 \mathrm{ml})$ was dispersed into an organic phase $(20 \mathrm{mg}$ PLGAPEG-PLGA dissolved in $5 \mathrm{ml}$ ethyl acetate) by vortex mixing, the $\mathrm{W} / \mathrm{O}$ emulsion was injected drop-by-drop into $50 \mathrm{ml} 2 \%$ PVA solution and underwent mechanical stirring $(1,000 \mathrm{rpm}$ for $5 \mathrm{~min}$ ). The resultant $\mathrm{W} / \mathrm{O} / \mathrm{W}$ emulsion was poured into $2 \%$ PVA aqueous solution $(450 \mathrm{ml})$. After then, the solution was stirred $(500 \mathrm{rpm}, 3 \mathrm{~h})$ to evaporate the organic solvent. The final microspheres were obtained after filtration, washing and freeze-drying. Urea-loaded microspheres (UM) were prepared in the same way as ULIM, except that urea-loaded liposome solution was replaced with urea solution $(0.5 \mathrm{ml}, 4 \mathrm{mg} / \mathrm{ml})$ as the water phase.

The following abbreviations are used: urea loaded liposomes (UL), urea-loaded microspheres (UM), and urea-loaded liposomes-in-microspheres (ULIM). Drug-free liposomes or microspheres are designated as blank liposomes or microspheres.

Size and zeta potential. The size and zeta potential were analyzed by a Zetasizer Nano S (Malvern Instruments Ltd., Malvern, UK). The size and distribution of microspheres were tested by a Malvern Mastersizer 2000 particle size analyzer (Malvern Instruments).

Drug encapsulation efficiency and loading of urea. The encapsulation efficacy and drug loading of urea in the formulations were determined as described below. After $0.2 \mathrm{ml}$ of 
the liposome solution was dissolved in methanol, the clear solution was analyzed by high performance liquid chromatography (HPLC L-2000; Hitachi). Alternatively, $5 \mathrm{mg}$ of microspheres were dissolved in $1 \mathrm{ml}$ dichloromethane. After dichloromethane was evaporated, $1 \mathrm{ml}$ methanol was added for HPLC analysis. A Hypersil $\mathrm{NH}_{2}$ column $(250$ x $4.6 \mathrm{~mm}$, $5 \mu \mathrm{m}$ ) was equipped in the HPLC, and the mobile phase was acetonitrile:water $(95: 5, \mathrm{v} / \mathrm{v})$. The flow rate was $1 \mathrm{ml} / \mathrm{min}$. The detection wavelength was $190 \mathrm{~nm}$. The column temperature was $25^{\circ} \mathrm{C}$. The encapsulation efficacy of urea $=$ the mass of encapsulated urea/the mass of total added urea $\mathrm{x} 100 \%$. The drug loading of urea $=$ the mass of encapsulated urea/the mass of liposomes or microspheres x $100 \%$.

In vitro drug release. Two milliliters liposomes or $10 \mathrm{mg}$ microspheres was transferred to a Spectra/Por ${ }^{\circledR}$ dialysis membrane (MWCO 1000). The sealed tube was put into a vial with $200 \mathrm{ml}$ phosphate-buffered saline (PBS) (pH 7.4, with or without $10 \% \mathrm{FBS})$. The vial was put in a water bath at $37^{\circ} \mathrm{C}$ with stirring (100 rpm). A total of $2 \mathrm{ml}$ of an aliquot of dialysate was taken out at different time-points. The concentration of urea was determined as described above.

CCK- 8 assays. HemECs (10,000/well) were seeded at subconfluency on a fibronectin-coated 96-well plate in EGM-2 with $10 \%$ FBS. Twelve hours later, the media were removed and the cells were treated with various concentrations of urea, liposomes or microspheres. After 72 or $120 \mathrm{~h}$, the cell viability was determined using the CCK- 8 kit according to the manual.

Cell treatments and transfections. HemECs (200,000/well) were seeded on a fibronectin-coated 6-well plate in EGM-2 with $10 \%$ FBS. Twelve hours later, the medium was removed, and the cells were treated with urea $(0,10,20$ and $50 \mu \mathrm{g} / \mathrm{ml})$ and incubated for a period of time (48, 72 or $96 \mathrm{~h})$. For gene knockdown or overexpression, specific siRNA (Santa Cruz Biotechnology, Santa Cruz, CA, USA) or recombinant lentiviral vectors (Shanghai GenePharma Co., Ltd., Shanghai, China) were transfected to the cells and incubated for $48 \mathrm{~h}$, before collection for analysis.

Western blot analysis. The cellular proteins were extracted, and separated by SDS-polyacrylamide gel electrophoresis. After the proteins were transferred onto polyvinylidene fluoride membranes, the membrane were incubated with the primary antibodies including anti-human HIF-1 $\alpha$ or VEGF and horseradish peroxidase conjugated to goat anti-mouse IgG as the secondary antibody (Santa Cruz Biotechnology). The GAPDH antibody (Santa Cruz Biotechnology) was used as the internal control antibody. The bands were detected with the Enhanced Chemiluminiscence kit (GE Healthcare) and visualized with the ChemiDoc XRS system (Bio-Rad Laboratories, Inc., Hercules, CA, USA).

Analysis of vascular endothelial growth factor (VEGF)-A. HemECs $(200,000 /$ well) were seeded on a fibronectin-coated 6 -well plate in EGM-2 with $10 \%$ FBS. Twelve hours later, the medium was removed and the cells were treated with various concentrations of the drugs. After a period of time, the VEGF-A concentration in the cellular supernatant was measured by
VEGF-A ELISA kits (R\&D Systems, Minneapolis, MN, USA) according to the manufacturer's instructions. In brief, $200 \mu \mathrm{l}$ of the sample was added to the plate and incubated for $2 \mathrm{~h}$. The sample was aspirated and washed. Afterwards, $200 \mu \mathrm{l}$ of conjugate was added and incubated for $2 \mathrm{~h}$. Finally, the substrate solution and stop solution was added sequentially. The absorbance was measured at 450/540 nm using a BioTek ELx800 Universal microplate reader.

Animal studies. The mice were purchased from the Shanghai Experimental Animal Center of the Chinese Academy of Sciences (Shanghai, China). All procedures were approved by the Committee on Animals of the Second Military Medical University (Shanghai, China) and all procedures were performed in accordance with the guidelines of the Committee on Animals of the Second Military Medical University (Shanghai, China).

A xenograft mouse model of $\mathrm{IH}$ was used to study the effects of urea on $\mathrm{IH}$ in vivo. HemECs $\left(1 \times 10^{7}\right)$ suspended in Matrigel were implanted subcutaneously into the flanks of female nude mice (6-8 weeks, $\sim 20 \mathrm{~g}$ ). After the hemangioma reached $\sim 25 \mathrm{~mm}^{3}$ on day 0 , mice were treated with single intratumoral (i.t.) injections of either formulation (UL, UM or ULIM, $2 \mathrm{mg}$ urea $/ \mathrm{kg}$ ), free urea (2 mg urea $/ \mathrm{kg}$ ) or blank LIM $(80 \mathrm{mg} / \mathrm{kg})$. The treatment was performed once every 5 days for 7 times (days 0 , 5, 10, 15, 20, 25 and 30). Propranolol was administered orally ( $2 \mathrm{mg} / \mathrm{kg}$, daily) for 30 days. Hemangioma was measured with a caliper, and the hemangioma volume $=\left(\right.$ width $^{2} \times$ length $) / 2$. The mice were euthanized on day 35 . The hemangioma was embedded in paraffin and stained with H\&E. The analysis of microvessel density (MVD) in the sections was performed as previously described (26).

Statistical analysis. Data were analyzed with the software SPSS 13.0 (SPSS, Inc., Chicago, IL, USA). A direct comparison between two groups was performed with the Student's non-paired t-test. One-way ANOVA with the Dunnett's or Newman Keuls post-test was used to compare the means of three or more groups. A $\mathrm{P}<0.05$ was considered statistically significant: ${ }^{*} \mathrm{P}<0.05 ;{ }^{* *} \mathrm{P}<0.01 ;{ }^{* * *} \mathrm{P}<0.001$; n.s. represents not significant $(\mathrm{P}>0.05)$.

\section{Results}

Preparation of urea-loaded liposomes-in-microspheres (ULIM). As described in Fig. 1, we firstly used the reverse evaporization method to develop urea-loaded liposomes (UL), and then encapsulated UL into microspheres to develop urea-loaded liposomes-in-microspheres (ULIM). We chose HSPC as the phospholipids in liposome preparation since it is saturated, neutral and has high phase transition temperature. Reverse vaporization was chosen to load urea in liposomes, since it is well regarded to encapsulate hydrophilic drugs in liposomes with high encapsulation efficacy.

Although the hydrophilic head groups protect liposomes, the liposomes could be readily damaged by the organic solvent used for the preparation of ULIM. Thus, chitosan was used to coat the liposomes to protect the liposomes-in-microspheres. Double emulsions are commonly used for encapsulating hydrophilic drugs suffering from low encapsulation efficiency 


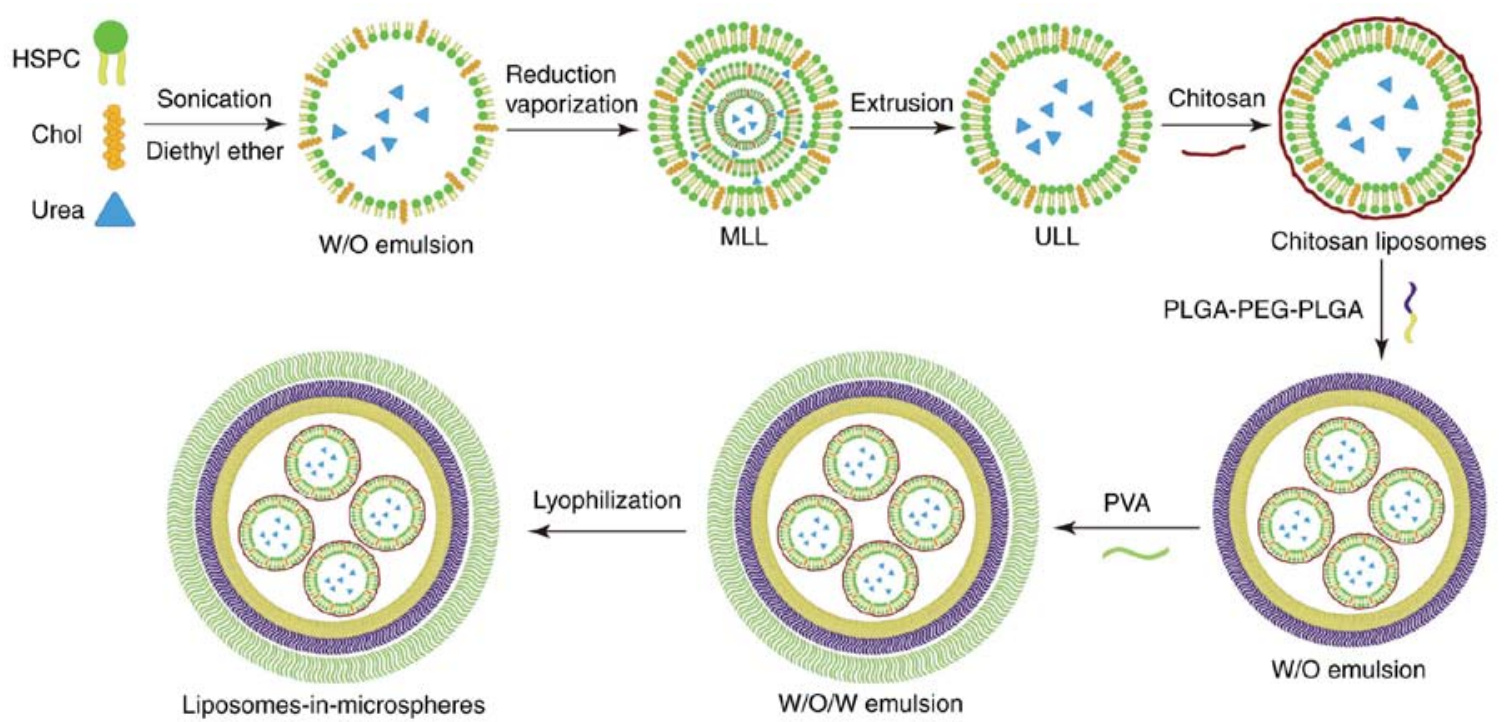

Figure 1. Preparation of urea-loaded liposomes and liposomes-in-microspheres. After specific amounts of lipids (HSPC and cholesterol) were dissolved in diethyl ether, urea solution was added to the lipid solution. The mixture was sonicated to stable $\mathrm{W} / \mathrm{O}$ emulsion. The diethyl ether was removed by reduction vaporization to form multilamellar liposomes (MLL). Unilamellar liposomes (ULL) were obtained by extruding the MLL with a membrane extruder Afterwards, the liposomes were coated with chitosan. The liposomes were dispersed into an organic phase consisting of PLGA-PEG-PLGA dissolved in ethyl acetate, resulting in a W/O emulsion. The W/O emulsion was injected drop-by-drop into PVA aqueous solution, and the resultant W/O/W emulsion was lyophilized to obtain urea-loaded liposomes-in-microspheres.
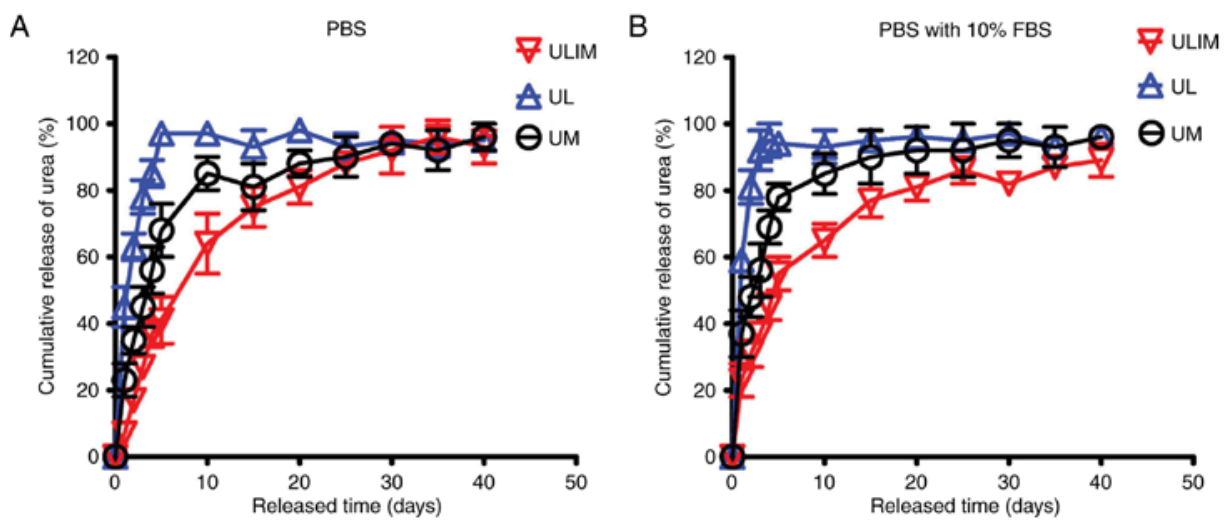

Figure 2. Drug release of liposomes and microspheres. A total of $2 \mathrm{ml}$ of liposome solution or $10 \mathrm{mg}$ microspheres were transferred to a dialysis membrane. The sealed tube was then introduced into a vial containing PBS with or without $10 \% \mathrm{FBS}$. The vial was secured in a water bath at $37^{\circ} \mathrm{C}$ with stirring. At predetermined time intervals, the amount of urea in the dialysate was determined by HPLC. (A) The in vitro release in $\mathrm{pH} 7.4 \mathrm{PBS}$. (B) The in vitro release in pH 7.4 PBS with 10\% FBS. Data are expressed as mean \pm SD (n=3). ULIM, urea-loaded liposomes-in-microspheres; UL, urea-loaded liposomes; UM, urealoaded microspheres.

Table I. Characterization of nanoparticles and microspheres. ${ }^{\mathrm{a}}$

\begin{tabular}{lcccrr}
\hline & Size $(\mathrm{nm} / \mu \mathrm{m})$ & Zeta potential $(\mathrm{mV})$ & PDI & EE $(\%)$ & Drug loading $(\%)$ \\
\hline UL & $186.3 \pm 24.7 \mathrm{~nm}$ & $-6.8 \pm 2.4$ & $0.16 \pm 0.04$ & $31.7 \pm 8.7$ & $10.3 \pm 3.1$ \\
ULIM & $62.4 \pm 21.3 \mu \mathrm{m}$ & - & - & $51.5 \pm 8.6$ & $2.5 \pm 0.5$ \\
$\mathrm{UM}$ & $53.8 \pm 25.9 \mu \mathrm{m}$ & - & - & $55.2 \pm 4.2$ & $3.4 \pm 2.7$ \\
\hline
\end{tabular}

${ }^{a}$ Data are expressed as mean $\pm \mathrm{SD}(\mathrm{n}=3)$ from three independent samples. UL, urea-loaded liposomes; UM, urea-loaded microspheres; ULIM, urea-loaded liposomes-in-microspheres. PDI, polydispersity; EE, encapsulation efficacy.

due to rapid drug partitioning into the external aqueous phase when using single emulsions. Thus, the double emulsion method was chosen to encapsulate hydrophilic liposomes in the microspheres. 

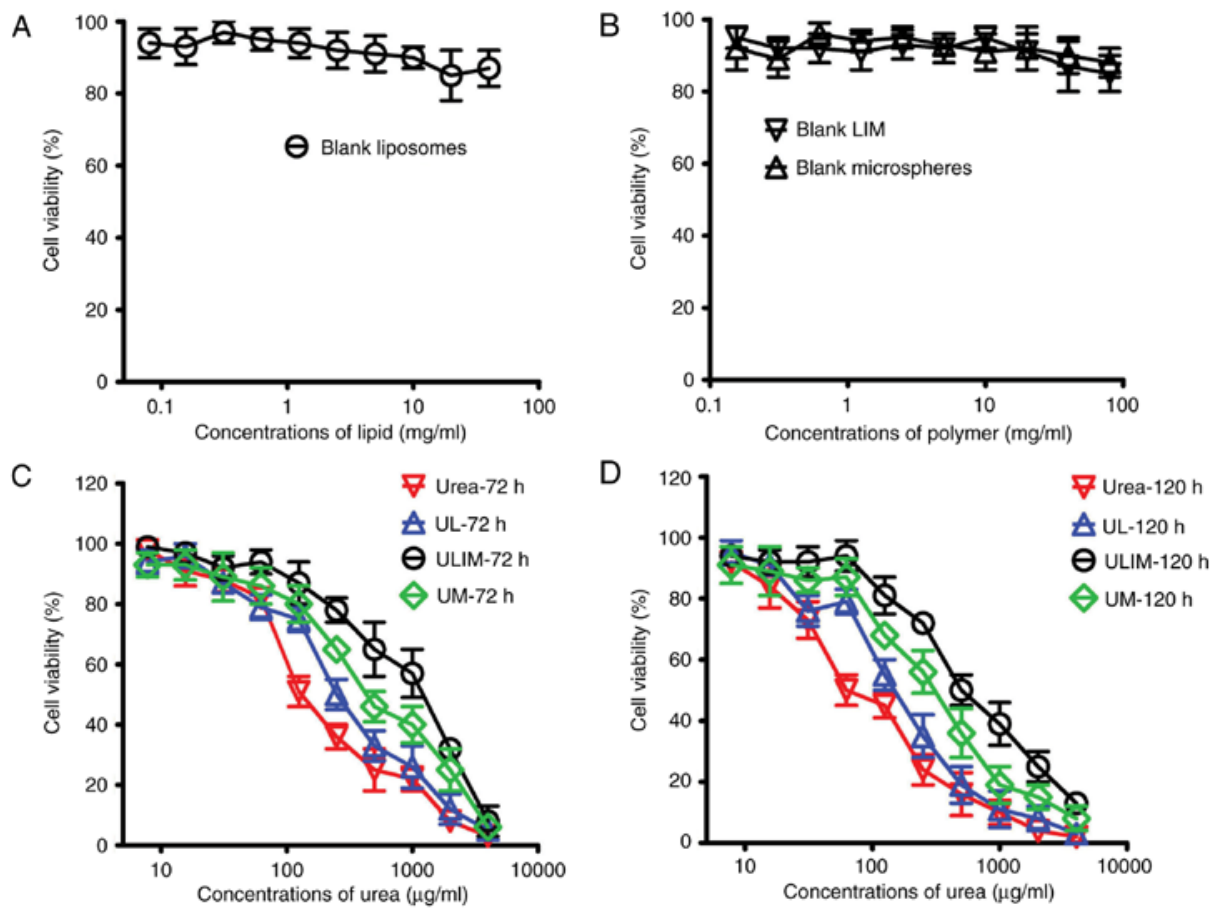

Figure 3. Dose-dependent cytotoxicity in HemECs at 72 and $120 \mathrm{~h}$. The cytotoxicity of (A) blank liposomes and (B) blank microspheres and LIM towards HemECs at $72 \mathrm{~h}$. The cytotoxicity of urea-loaded formulations towards HemECs at (C) $72 \mathrm{~h}$ and (D) $120 \mathrm{~h}$. The cell viability was evaluated by the CCK-8 assay. Data are expressed as mean $\pm \mathrm{SD}(\mathrm{n}=3)$. ULIM, urea-loaded liposomes-in-microspheres; UL, urea-loaded liposomes; UM, urea-loaded microspheres; LIM, liposomes-in-microspheres

Table II. $\mathrm{IC}_{50}$ values in HemECs following 72 and $120 \mathrm{~h}$ of treatment.

\begin{tabular}{lcc}
\hline $\mathrm{IC}_{50}, \mu \mathrm{g} / \mathrm{ml}$ & $72 \mathrm{~h}$ & $120 \mathrm{~h}$ \\
\hline Urea & $168 \pm 29$ & $98 \pm 21$ \\
UL & $218 \pm 36$ & $133 \pm 32$ \\
UM & $502 \pm 52$ & $389 \pm 76$ \\
ULIM & $1238 \pm 252$ & $631 \pm 139$
\end{tabular}

${ }^{a}$ Data are expressed as mean $\pm \mathrm{SD}(\mathrm{n}=3)$. UL, urea-loaded liposomes; UM, urea-loaded microspheres; ULIM, urea-loaded liposomes-inmicrospheres.

Characteristics of UL and ULIM. Table I summarizes the characteristics of the liposomes and microspheres. The size of UL was $186 \mathrm{~nm}$, with a low PDI of 0.16 (suggesting a homogeneous size distribution). UL showed a negative zeta potential of $-6.8 \mathrm{mV}$. The encapsulation efficacy (EE) of urea in UL is $31.7 \%$, and the drug loading of UL is $10.3 \%$, suggesting that reverse vaporization is a suitable approach to encapsulate urea in liposomes. ULIM have a size of $62.4 \mu \mathrm{m}$, which is similar to that of UM $(53.8 \mu \mathrm{m})$. The EE of urea in ULIM is $51.5 \%$, which is similar to that of UM (55.2\%).

The drug release profiles of UL, UM and ULIM were evaluated in PBS and PBS with 10\% FBS. In PBS, UL showed a quick urea release $(45 \%$ of urea was released at day 1 , and $85 \%$ at day 4) (Fig. 2A). The reason why UL quickly released urea is that hydrophilic drugs tend to permeate across the liposomal membrane quickly (19). In contrast, UM showed a reduced release (23\% of urea was released at day 1 and $56 \%$ at day 4). Significantly, ULIM showed the slowest release (only $7 \%$ of urea was released at day 1 and $37 \%$ at day 4). It took 4,10 and 20 days for UL, UM and ULIM to release $>80 \%$ of urea, respectively.

Similar results were obtained in the urea release in PBS with $10 \%$ FBS. UL showed a quick urea release (59\% of urea was released at day 1 and 95\% at day 4) (Fig. 2B). In contrast, UM showed a reduced release ( $37 \%$ of urea was released at day 1 and $69 \%$ at day 4). Significantly, ULIM showed the slowest release (only $23 \%$ of urea was released at day 1 and $45 \%$ at day 4). It took 2, 10 and 20 days for UL, UM and ULIM to release $>80 \%$ of urea, respectively.

Thus, the urea release was significantly reduced in ULIM compared with UL and UM, suggesting that encapsulation of urea-loaded liposomes-in-microspheres significantly retard the release of urea from liposomes or microspheres.

Cytotoxicity towards HemECs. To assess the biocompatibility of our prepared formulations, we evaluated the cytotoxicity of the blank liposomes, microspheres and LIM. As shown in Fig. 3A and B, all the blank formulations showed little toxicity to HemECs (reflected by the cell viability which exceeded $85 \%)$.

On the contrary, urea, UL, UM and ULIM showed a dosedependent cytotoxicity towards HemECs (Fig. 3C and D). Their $\mathrm{IC}_{50}$ values are shown in Table II. The $72 \mathrm{~h} \mathrm{IC}$ 50 values of urea, UL, UM and ULIM were found to be 168, 218, 502 and $1238 \mu \mathrm{g} / \mathrm{ml}$, respectively, suggesting that the cytotoxic effect of urea was significantly decreased when urea is loaded in microspheres or liposomes-in-microspheres. Similar results were obtained in the $120 \mathrm{~h} \mathrm{IC}_{50}$ values. The $120 \mathrm{~h} \mathrm{IC}_{50}$ values 

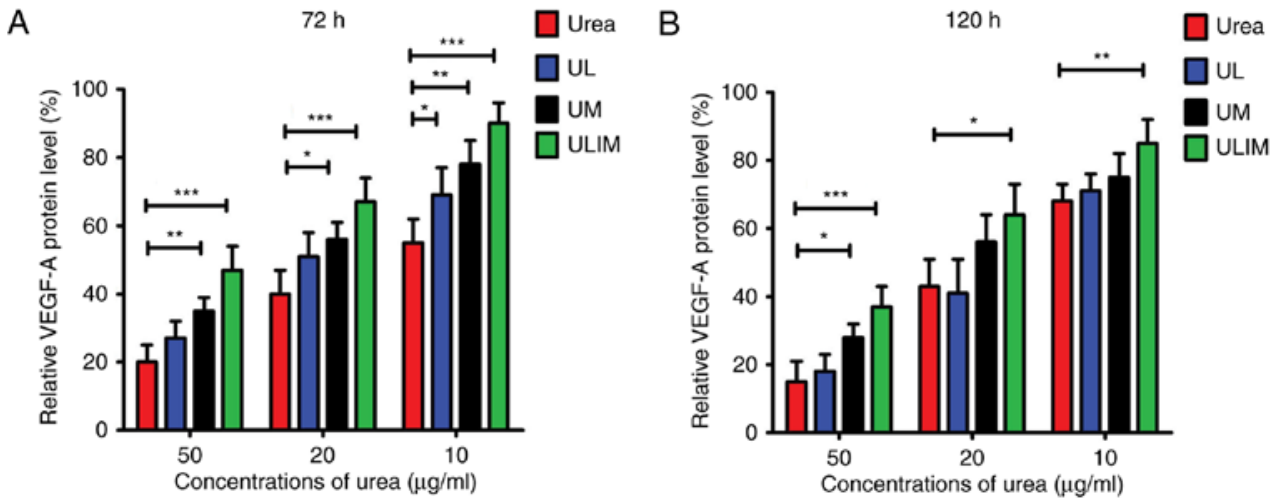

Figure 4. VEGF-A expression level in HemECs after treatment. After HemECs were seeded on a fibronectin-coated 6-well plate, the cells were treated with various concentrations of urea-loaded formulations for 72 or $120 \mathrm{~h}$. The VEGF-A concentration of the cellular supernatant was measured by VEGF-A ELISA kits. The relative protein level was expressed as the percentage of the protein of the treated groups relative to the untreated group. The relative protein level of the urea-treated group was compared with other groups by one-way ANOVA with the Dunnett's post-test. ${ }^{*} \mathrm{P}<0.05 ;{ }^{* *} \mathrm{P}<0.01 ;{ }^{* * *} \mathrm{P}<0.001$. Data are expressed as mean $\pm \mathrm{SD}(\mathrm{n}=3)$. UL, urea-loaded liposomes; UM, urea-loaded microspheres; ULIM, urea-loaded liposomes-in-microspheres.
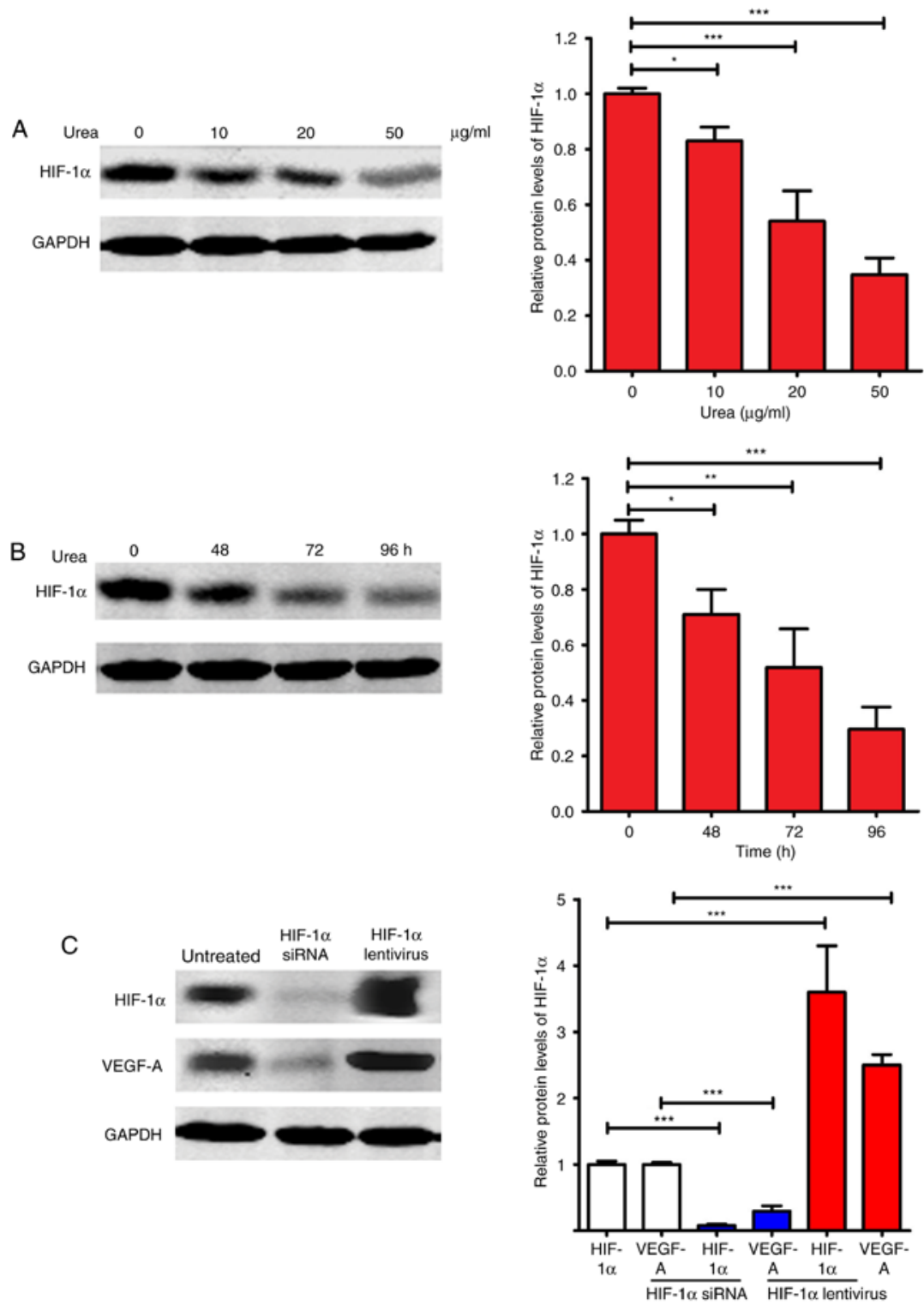

Figure 5. Effect of urea on the expression of HIF-1 $\alpha$ in HemECs in vitro. (A) Western blot analysis of HIF-1 $\alpha$ protein expression in cells treated with different doses of urea for $72 \mathrm{~h}$. (B) Western blot analysis of HIF-1 $\alpha$ protein expression in cells treated with $20 \mu \mathrm{g} / \mathrm{ml}$ of urea for different time periods. (C) Expression of HIF-1 $\alpha$ or VEGF-A after transfection of HIF-1 $\alpha$ siRNA or HIF-1 $\alpha$ lentivirus. siRNA or lentiviral vectors were transfected to the cells and incubated for $48 \mathrm{~h}$, before collection for analysis. The quantification of relative protein levels are shown on the right of each panel. The relative protein level of the control group was compared with other groups by one-way ANOVA with the Dunnett's post-test. ${ }^{*} \mathrm{P}<0.05 ;{ }^{* *} \mathrm{P}<0.01 ;{ }^{* * *} \mathrm{P}<0.001$. Data are expressed as mean $\pm \mathrm{SD}(\mathrm{n}=3)$. 
A

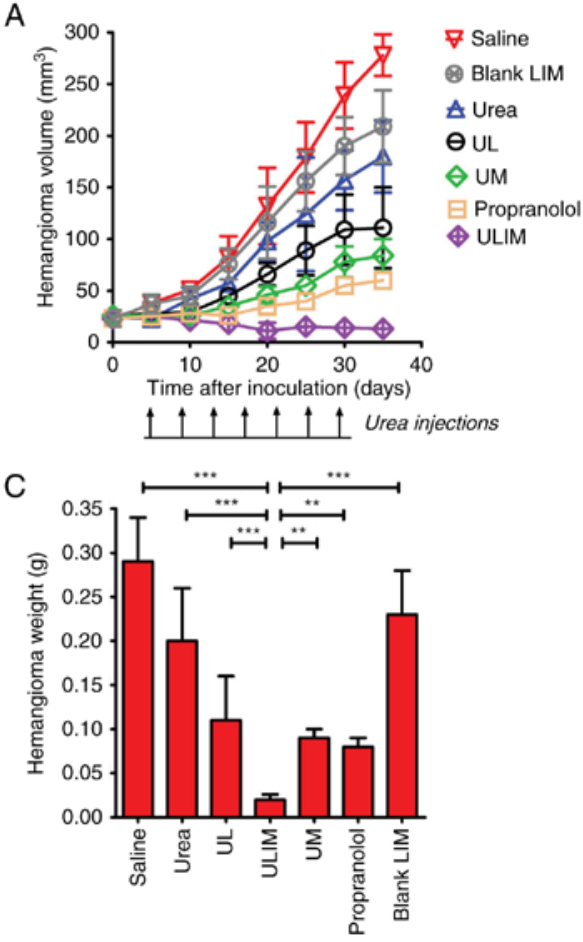

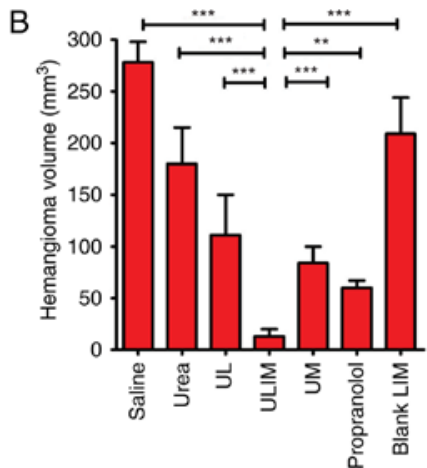

D

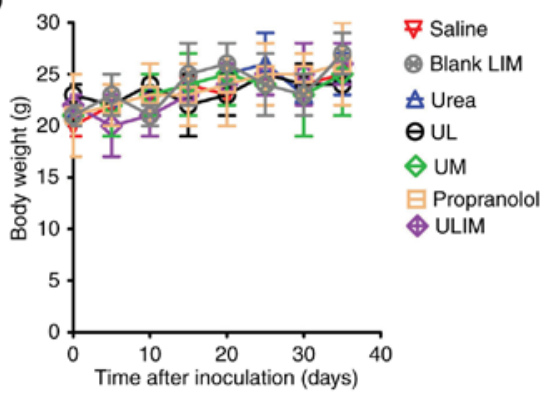

Figure 6. Therapeutic effect of urea in mice bearing subcutaneous IH xenografts. When the hemangiomas had reached $\sim 25 \mathrm{~mm}^{3}$ in size (day 0 ), mice were treated with single intratumoral injections of either formulation (UL, UM and ULIM, $2 \mathrm{mg} \mathrm{urea} / \mathrm{kg}$ ), free urea (2 mg urea/kg) or blank LIM (80 mg/kg) Propranolol was administrated orally ( $2 \mathrm{mg} / \mathrm{kg}$, daily) for 30 days. Treatments were carried out on days $0,5,10,15,20,25$ and 30 (indicated by black arrows). (A) The emangioma growth curve. (B) Hemangioma volume at the end point (day 35). (C) The excised tumors were weighed at the end point. The tumor volume or weight of the ULIM-treated group was compared with that of other groups by one-way ANOVA with the Dunnett's post-test. ${ }^{*} \mathrm{P}<0.05 ;{ }^{* *} \mathrm{P}<0.01$; ${ }^{* * *} \mathrm{P}<0.001$. (D) The weight change of the mice during the treatment. The body weight of the mice was monitored once every five days. Data are expressed as mean \pm SD $(\mathrm{n}=8)$. UL, urea-loaded liposomes; UM, urea-loaded microspheres; ULIM, urea-loaded liposomes-in-microspheres.

of urea, UL, UM and ULIM were found to be $98,133,389$ and $631 \mu \mathrm{g} / \mathrm{ml}$, respectively. We speculate that the significantly reduced cytotoxic effects of UM and ULIM are attributed to their slow release of urea.

VEGF-A expression level in HemECs after treatment. The heparin-binding growth factor VEGF-A is able to induce angiogenesis (27). Fig. 4A shows that urea, UL, UM and ULIM exerted dose-dependent inhibition of VEGF-A protein expression in HemECs at $72 \mathrm{~h}$. At the concentrations of urea ranging from 10 to $50 \mu \mathrm{g} / \mathrm{ml}$, urea was more efficient than UM and ULIM in the inhibition of VEGF-A expression ( $\mathrm{P}<0.05$ for $\mathrm{UM}, \mathrm{P}<0.001$ for ULIM). Urea at $50 \mu \mathrm{g} / \mathrm{ml}$ inhibited VEGF-A expression by $\sim 80 \%$, whereas ULIM only inhibited by $\sim 60 \%$. Consistently, urea was more effective at inhibiting VEGF-A expression than ULIM at the concentrations of urea ranging from 10 to $50 \mu \mathrm{g} / \mathrm{ml}$ at $120 \mathrm{~h}(\mathrm{P}<0.05$; Fig. 4B). At $50 \mu \mathrm{g} / \mathrm{ml}$, urea inhibited VEGF-A expression by $>80 \%$, whereas ULIM inhibited VEGF-A expression by $\sim 60 \%$. Taken together, urea was more effective in inhibiting both VEGF-A expression than ULIM. We speculate that the significant reduced activity of ULIM against inhibition of VEGF-A expression is attributed to its slow release of urea.

HIF-1 $\alpha$ expression level in HemECs after treatment. Hypoxiainducible factor- $1 \alpha$ (HIF-1 $\alpha)$ and VEGF-A are critical factors in promoting angiogenesis $(28,29)$. HIF- $1 \alpha$ is a master regulator of VEGF-A, and could activate transcription of the VEGF-A gene (29). We evaluated the expression of HIF-1 $\alpha$ after urea treatment. As shown in Fig. 5A and B, urea inhibited the expression of HIF-1 $\alpha$ in a dose- and time-dependent manner. At $50 \mu \mathrm{g} / \mathrm{ml}$, urea inhibited HIF-1 $\alpha$ expression by $\sim 70 \%$ relative to the untreated control $(\mathrm{P}<0.001)$. At $96 \mathrm{~h}$, urea at $20 \mu \mathrm{g} / \mathrm{ml}$ inhibited HIF-1 $\alpha$ expression by $\sim 75 \%$ relative to the untreated control $(\mathrm{P}<0.001)$. Since the expression of VEGF-A is directly regulated by $\mathrm{HIF}-1 \alpha$, we investigated whether regulated expression of $\mathrm{HIF}-1 \alpha$ affects VEGF-A expression (Fig. 5C). After the expression of HIF-1 $\alpha$ was knocked down by HIF-1 $\alpha$ siRNA, the expression of VEGF-A was significantly repressed $(\mathrm{P}<0.001)$. In contrast, the expression of VEGF-A was significantly upregulated $(\mathrm{P}<0.001)$ after the overexpression of HIF-1 $\alpha$ by transfection of HIF-1 $\alpha$ lentivirus. Taken together, these results suggested that the downregulated VEGF-A expression after urea treatment was at least partially caused by the urea-induced inhibition of HIF-1 $\alpha$ expression.

Inhibition of subcutaneous hemangioma growth in vivo. The therapeutic effect of the various formulations was examined in mice bearing subcutaneous hemangioma. As shown in Fig. 6A, on day 35, blank liposomes-in-microspheres (LIM) did not show significant antitumor activity, and the blank LIM-treated hemangioma progressed rapidly. As expected, oral administration of propranolol achieved superior therapeutic efficacy, and resulted in a $78 \%$ decrease in hemangioma volume. Notably, ULIM treatment resulted in a $95 \%$ decrease in hemangioma volume, whereas UM, UL and urea treatment only resulted in a 70, 60 and $35 \%$ decrease, respectively. At the end point, compared with the initial volume $\left(25 \mathrm{~mm}^{3}\right)$, 


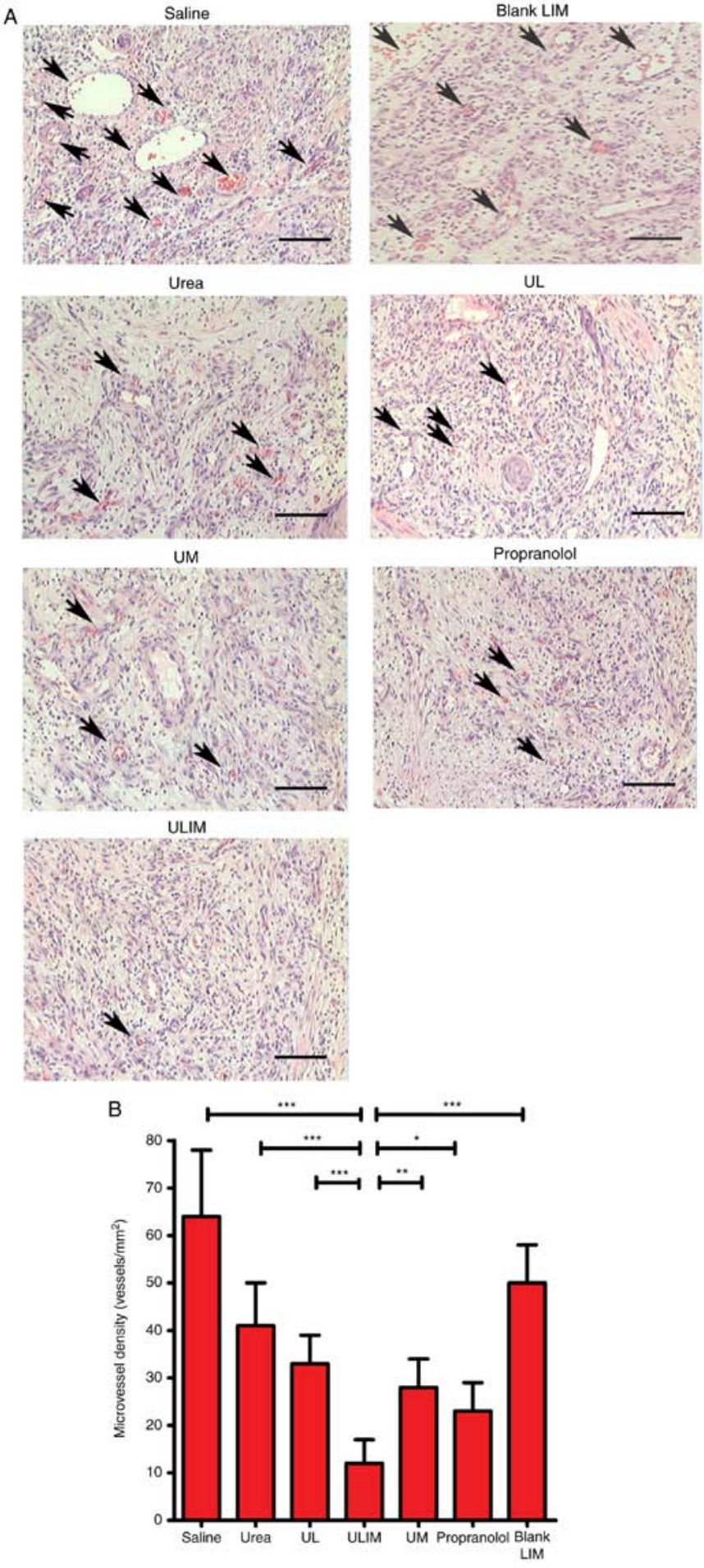

Figure 7. The therapeutic effect of urea in mice bearing subcutaneous $\mathrm{IH}$ xenografts, as reflected by the H\&E staining of sections and microvessel density (MVD) analysis of the sections. On day 35, the mice were euthanized. The hemangiomas were collected, fixed and embedded in paraffin for histological analysis. (A) H\&E staining of sections. Black arrows indicated lumens with red blood cells. Scale bars represent $100 \mu \mathrm{m}$. (B) MVD quantification of hemangioma. Lumens with red blood cells (A, arrows) were counted. Data are expressed as mean $\pm \mathrm{SD}(\mathrm{n}=8) .{ }^{*} \mathrm{P}<0.05 ;{ }^{* *} \mathrm{P}<0.01 ;{ }^{* * *} \mathrm{P}<0.001$. UL, urea-loaded liposomes; UM, urea-loaded microspheres; ULIM, urea-loaded liposomes-in-microspheres.

the hemangioma volume in the propranolol-treated mice had increased by 2.4 -fold, whereas the hemangioma volume in the ULIM-treated mice did not increase but gradually decreased.
The hemangioma volume of the ULIM-treated group was significantly smaller than that of other groups (saline, $278 \mathrm{~mm}^{3}$; blank LIM, $209 \mathrm{~mm}^{3}$; urea, $180 \mathrm{~mm}^{3}$; UL, $111 \mathrm{~mm}^{3}$; UM, $84 \mathrm{~mm}^{3}$; ULIM, $13 \mathrm{~mm}^{3}$; ULIM vs. saline: $\mathrm{P}<0.001$; ULIM vs. urea, $\mathrm{P}<0.001$; ULIM vs. UL, $\mathrm{P}<0.001$; ULIM vs. $\mathrm{UM}, \mathrm{P}<0.001$; ULIM vs. propranolol, $\mathrm{P}<0.01$; ULIM vs. blank LIM, P<0.001) (Fig. 6B).

The hemangioma was weighed at the endpoint (Fig. 6C). The mean hemangioma weight of the ULIM-treated group was significantly lower than that of other groups (saline, $0.28 \mathrm{~g}$; urea, $0.20 \mathrm{~g}$; UL, $0.11 \mathrm{~g}$; ULIM, $0.02 \mathrm{~g}$; UM, $0.09 \mathrm{~g}$; propranolol, $0.08 \mathrm{~g}$; blank LIM, $0.23 \mathrm{~g}$; ULIM vs. saline, $\mathrm{P}<0.001$; ULIM vs. urea, $\mathrm{P}<0.001$; ULIM vs. $\mathrm{UL}, \mathrm{P}<0.001$; ULIM vs. UM, $\mathrm{P}<0.01$; ULIM vs propranolol, $\mathrm{P}<0.05$; ULIM vs. blank LIM, $\mathrm{P}<0.001)$.

The toxicity of all treatments was measured by observing any physical or behavioral changes post treatment and by monitoring the weight of mice. In the urea-treated group, skin irritation, as reflected by the red and swollen skin, was observed in some mice, but all cases of skin irritation disappeared spontaneously in 3 days and need no special treatments. None of the treated mice showed any behavioral changes. None of the treated mice showed significant change in weight compared to the saline control (Fig. 6D). Taken together, the results showed that all the treatments were well-tolerated by mice bearing hemangioma.

The hemangiomas excised from the mice were stained with hematoxylin and eosin (H\&E) (Fig. 7A), and microvessel density (MVD) analysis of the histological sections was performed (Fig. 7B). The mean MVD of the ULIM-treated group was significantly lower than that of other groups (saline, 64 vessels $/ \mathrm{mm}^{2}$; urea, 41 vessels $/ \mathrm{mm}^{2}$; UL, 33 vessels $/ \mathrm{mm}^{2}$; ULIM, 12 vessels $/ \mathrm{mm}^{2}$; UM, 28 vessels $/ \mathrm{mm}^{2}$; propranolol, 23 vessels $/ \mathrm{mm}^{2}$; blank LIM, 50 vessels $/ \mathrm{mm}^{2}$; ULIM vs. saline: $\mathrm{P}<0.001$, ULIM vs. urea: $\mathrm{P}<0.001$; ULIM vs. UL: $\mathrm{P}<0.001$; ULIM vs. UM: $\mathrm{P}<0.01$; ULIM vs. propranolol: $\mathrm{P}<0.05$; ULIM vs. blank LIM: $\mathrm{P}<0.001)$, suggesting that ULIM was the most effective at inhibiting the vascularization of hemangioma among all the groups.

\section{Discussion}

$\mathrm{IH}$ is a benign pediatric tumor, and rapid growth of IH can result in serious morbidity and even mortality. We demonstrated that urea is an effective and well-tolerated treatment for IH (8-11). To reduce the daily administration of urea in the treatment of IH, we firstly utilized ULIM as a topical controlled release system to realize the sustained release of urea. This study demonstrated that ULIM achieved superior therapeutic efficacy compared with urea and propranolol, and reduced the daily administration frequency of urea.

The selection of an anti-hemangioma is critically important for the superior activity of our prepared ULIM. Urea is an organic compound and a normal body metabolite, and is widely used in various skin diseases $(5,6)$. Importantly, urea has been used in the treatment of IH in China since 1970s (7). Our hospital, Henan Provincial People's Hospital (Zhengzhou, China), has used urea in the treatment of IH for 20 years, and we have demonstrated that urea is an effective drug for the treatment of IH with few side-effects (8-11). Thus, the superior 


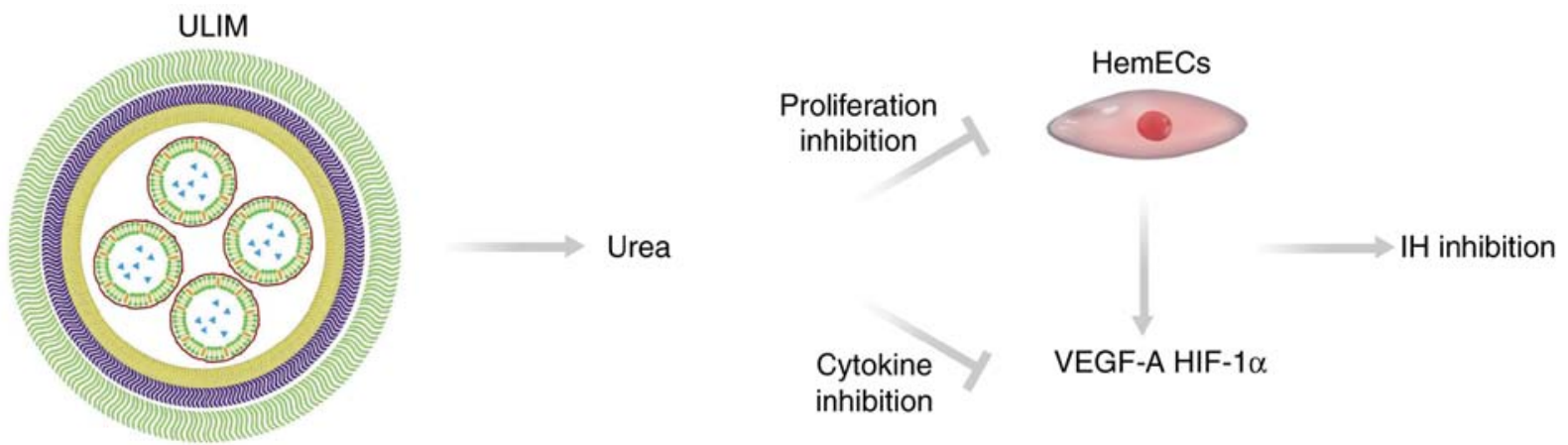

Figure 8. Mechanism underlying the anti-hemangioma activity of ULIM. After being released from the ULIM, urea inhibits the proliferation of HemECs, and the production of angiogenesis factors including VEGF-A and HIF-1 $\alpha$, resulting in inhibition of IH growth. ULIM, urea-loaded liposomes-in-microspheres.

effectiveness and few side-effects of urea, coupled with the immediate availability of the medication, led to a rapid and wide use of urea for IH. As should be, the application of urea for IH will be more convenient if its frequency of administration could be reduced.

Changing the route of administration could enhance the therapeutic efficacy and reduce the side-effects of drugs. To overcome the high frequency of urea administration, we developed a practical sustained release system defined as ULIM to release urea. The data presented here confirmed that ULIM showed a significantly slower release of urea, compared with UL and UM, as reflected by the fact that it took 4, 10 and 20 days for UL, UM and ULIM to release $>80 \%$ of urea, respectively. Feng et al (22) elucidated the mechanism underlying the drug release from liposomes-in-microspheres. First, liposomes must diffuse through tortuous water channels of microspheres. As time passes, the degradation of the polymer matrix causes expansion of the tortuous water channels, thereby leading to a sustained release of liposomes. When the liposomes are released, they still act as a sustained release reservoir of drugs. Thus, the mechanism underlying the significantly slower release of urea by ULIM compared with UL and UM could be clarified as follows. Since liposomes are soft and easily ruptured, the release of urea from UL is quick. PLGA microspheres which possess a more rigid structure show relatively slower urea release after the gradual degradation of the polymer matrix. As regards as ULIM, the release of urea undergoes two stages. UL must slowly diffuse through tortuous water channels of microspheres. After UL is released from ULIM, UL still serves as a reservoir of urea and urea is gradually released from UL. Thus, urea is released at a very slow rate from ULIM since it needs to conquer two barriers consisting of the liposome membrane and the PLGA polymer matrix.

Although ULIM were less efficient in the inhibition of the proliferation of HemECs and VEGF expression than urea, UL and UM in vitro, its therapeutic effect was the best in vivo, as reflected by significantly reduced hemangioma volume, weight and MVD. Notably, ULIM were superior to daily administration of propranolol which is the only FDA approved drug for the treatment of $\mathrm{IH}$. We speculate that the discrepancy of ULIM could be clarified as follows. When tested in vitro, all the drugs are restricted to cell culture plates, and the activity of urea depends on the concentration of urea released from the formulations. The slow release of urea from ULIM results in a reduced cytotoxic effect, compared with urea, UL and UM. The quick release of urea from UL will surely induce superior cytotoxic effects. However, after intratumor injection in vivo, urea and UL undergo quick elimination of urea from hemangioma, resulting in poor therapeutic efficacy for hemangioma, whereas the prolonged and sustained release of urea from ULIM and UM significantly retard the angiogenesis of hemangioma.

We investigated the effect of urea on the expression of VEGF-A and HIF-1 $\alpha$ which promote angiogenesis $(28,29)$. It is noteworthy that urea significantly repressed the expression of VEGF-A and HIF- $1 \alpha$ in a dose-dependent manner. Since HIF-1 $\alpha$ is a master regulator of VEGF-A, and activates transcription of the VEGF-A gene (29), we investigated whether regulated expression of HIF-1 $\alpha$ affects VEGF-A expression. VEGF-A expression was significantly repressed after HIF-1 $\alpha$ was knocked down, whereas VEGF-A expression was upregulated after HIF-1 $\alpha$ overexpression, suggesting that downregulated VEGF-A expression after urea treatment was at least partially caused by the urea-induced inhibition of HIF-1 $\alpha$ expression. Propranolol has been also reported to be able to inhibit IH by suppressing VEGF-A in an HIF-1 $\alpha$ dependent manner (30).

The safety of a drug delivery systems is important in the clinic (31). The components of our prepared ULIM include liposomes, chitosan and PLGA, which are rather biocompatible materials. Furthermore, our data revealed that none of the treated mice showed behavioral changes, severe side-effects and weight loss. Thus, the safety of ULIM should be superior in clinical use. The detailed in vivo distribution and further safety data of ULIM should be investigated in further studies. Furthermore, the intratumor injection approach of our microspheres is safe in the clinic. Based on our 10-year clinical experience for $\mathrm{IH}$, we can safely conclude that needle injection into hemangioma does not induce bleeding even in large hemangiomas, and the slight bleeding that occurs can be easily suppressed by hand compression.

Our results elucidated the mechanism of the anti-hemangioma activity of ULIM (Fig. 8). ULIM tends to accumulate in IH after intratumor injection. After being released from ULIM urea inhibits the proliferation of HemECs and reduces expression of angiogenesis factors including VEGF-A and HIF-1 $\alpha$. The reduced expression of VEGF-A and HIF-1 $\alpha$ would also 
significantly retard the angiogenesis in IH. On the contrary, when free urea is injected into IH, it is eliminated quickly by the blood circulation, resulting in poor therapeutic efficacy of $\mathrm{IH}$.

In conclusion, we demonstrated that urea is an effective and well-tolerated treatment of IH, whereas its frequent administration reduces the compliance of patients. We hereby firstly utilized ULIM as a topical controlled release system to realize the sustained release of urea. ULIM have been demonstrated to show sustained release of urea, achieving superior therapeutic efficacy compared with urea and propranolol, and significantly reducing the administration frequency of urea. Moreover, the safety of ULIM is rather promising. Thus, our findings show that ULIM is a promising treatment for IH.

\section{Acknowledgements}

The present study was supported by the Henan Medical Provincial Science and Technology Plan Project (project number: 142102310080 and 201702202).

\section{References}

1. Kilcline $\mathrm{C}$ and Frieden IJ: Infantile hemangiomas: how common are they? A systematic review of the medical literature. Pediatr Dermatol 25: 168-173, 2008.

2. Chen TS, Eichenfield LF and Friedlander SF: Infantile hemangiomas: an update on pathogenesis and therapy. Pediatrics 131: 99-108, 2013.

3. Castaneda S, Melendez-Lopez S, Garcia E, De la Cruz H and Sanchez-Palacio J: The role of the pharmacist in the treatment of patients with infantile hemangiomas using propranolol. Adv Ther 33: 1831-1839, 2016.

4. Khorsand K, Backus S and Sidbury R: What's new in pediatric dermatology. Current Dermatology Reports 3: 187-190, 2014.

5. Long CL, Jeevanandam M and Kinney JM: Metabolism and recycling of urea in man. Am J Clin Nutr 31: 1367-1382, 1978.

6. Fredriksson T and Gip L: Urea creams in the treatment of dry skin and hand dermatitis. Int J Dermatol 14: 442-444, 1975.

7. Li G and Gu J: Infantile Hemangioma. 1st edition. Shaanxi Science and Technology Press, Xian 1991 (In Chinese).

8. Liang X, Dong C, Ma Y, Lei H, Liu D, Qiao J and Xiao L: The treatment of spongy infantile hemangioma on body surface by local urea injection. Chin Hosp Pharm J 31: 1396-1397, 2011 (In Chinese).

9. Chang Y, Dong $\mathrm{C}$ and Zheng W: The clinical observation of 80 cases of infantile hemangioma by urea local injection. Clin Med 19: 52-53, 1999 (In Chinese).

10. Lei H, Huang J, Meng X, Zhang W, Dong C, Sun B and Ma Y: Carbonyldiamide injection therapy for 167 cases of infant hemangiomas at peculiar region. J Medical Forum 35: 3-5, 2014 (In Chinese).

11. Guo X, Zhu X, Dong C and Ma Y: Clinical analysis of 32 cases of vulvar sponge hemangioma receiving intratumoral urea injection combined with surgery. Chin J Obstet Gynecol 50: 221-223, 2015 (In Chinese).

12. Müller-Goymann CC: Physicochemical characterization of colloidal drug delivery systems such as reverse micelles, vesicles, liquid crystals and nanoparticles for topical administration. Eur J Pharm Biopharm 58: 343-356, 2004.
13. Almeida H, Amaral MH, Lobao P, Frigerio C and Sousa Lobo JM: Nanoparticles in ocular drug delivery systems for topical administration: promises and challenges. Curr Pharm Des 21: 5212-5224, 2015.

14. Freiberg $S$ and Zhu XX: Polymer microspheres for controlled drug release. Int J Pharm 282: 1-18, 2004.

15. Gao J, Xia Y, Chen H, Yu Y, Song J, Li W, Qian W, Wang H, Dai J and Guo Y: Polymer-lipid hybrid nanoparticles conjugated anti-EGFR antibody for targeted drug delivery to hepatocellular carcinoma. Nanomedicine (Lond) 9: 279-293, 2014.

16. Van Slooten ML, Boerman O, Romøren K, Kedar E, Crommelin DJ and Storm G: Liposomes as sustained release system for human interferon-gamma: biopharmaceutical aspects. Biochim Biophys Acta 1530: 134-145, 2001

17. Xiao C, Qi X, Maitani Y and Nagai T: Sustained release of cisplatin from multivesicular liposomes: potentiation of antitumor efficacy against S180 murine carcinoma. J Pharm Sci 93: 1718-1724, 2004

18. Bittman R and Clejan S: Kinetics of cholesterol and phospholipid exchange between mycoplasma membranes and lipid vesicles. Isr J Med Sci 23: 398-402, 1987.

19. New RRC: Liposomes: A Practical Approach. Publisher: Oxford University Press. Revised edition, 1990.

20. Shive MS and Anderson JM: Biodegradation and biocompatibility of PLA and PLGA microspheres. Adv Drug Deliv Rev 28: 5-24, 1997.

21. Dass CR, Walker TL, Kalle WH and Burton MA: A microsphereliposome (microplex) vector for targeted gene therapy of cancer. II. In vivo biodistribution study in a solid tumor model. Drug Deliv 7: 15-9, 2000.

22. Feng SS, Ruan G and Li QT: Fabrication and characterizations of a novel drug delivery device liposomes-in-microspheres (LIM). Biomaterials 25: 5181-5189, 2004.

23. Medici D and Olsen BR: Rapamycin inhibits proliferation of hemangioma endothelial cells by reducing HIF-1-dependent expression of VEGF. PLoS One 7: e42913, 2012.

24. Shuming C, Shaoquan C, Zaizhong Z, Chengjin L, Yin X, Chen L, Qingjin $\mathrm{H}$ and Lie W: Effects of topical rapamycin hydrochloride gel for treatment of infantile hemangiomas in nude mice). Zhonghua Zheng Xing Wai Ke Za Zhi 31: 446-450, 2015 (In Chinese)

25. Wang Z, Li J, Xu X, Duan X and Cao G: Urea immunoliposome inhibits human vascular endothelial cell proliferation for hemangioma treatment. World J Surg Oncol 11: 300, 2013.

26. Greenberger S, Yuan S, Walsh LA, Boscolo E, Kang KT, Matthews B, Mulliken JB and Bischoff J: Rapamycin suppresses self-renewal and vasculogenic potential of stem cells isolated from infantile hemangioma. J Invest Dermatol 131: 2467-2476, 2011.

27. Mason JC, Lidington EA, Ahmad SR and Haskard DO: bFGF and VEGF synergistically enhance endothelial cytoprotection via decay-accelerating factor induction. Am J Physiol Cell Physiol 282: 578-587, 2002.

28. Manalo DJ, Rowan A, Lavoie T, Natarajan L, Kelly BD, Ye SQ, Garcia JG and Semenza GL: Transcriptional regulation of vascular endothelial cell responses to hypoxia by HIF-1. Blood 105: 659-669, 2005.

29. Déry MA, Michaud MD and Richard DE: Hypoxia-inducible factor 1: regulation by hypoxic and non-hypoxic activators. Int J Biochem Cell Biol 37: 535-540, 2005.

30. Li P, Guo Z, Gao Y and Pan W: Propranolol represses infantile hemangioma cell growth through the $\beta 2$-adrenergic receptor in a HIF-1 $\alpha$-dependent manner. Oncol Rep 33: 3099-3107, 2015.

31. Nystrom AM and Fadeel B: Safety assessment of nanomaterials: implications for nanomedicine. J Control Release 161: 403-408, 2012.

This work is licensed under a Creative Commons Attribution-NonCommercial-NoDerivatives 4.0 International (CC BY-NC-ND 4.0) License. 